\title{
A Review of Acoustical Methods for Porous Material Characterisation
}

\author{
Kirill V. Horoshenkov \\ Department of Mechanical Engineering, University of Sheffield, S1 3JD, United Kingdom.
}

(Received 25 October 2015; accepted 24 June 2016)

\begin{abstract}
The purpose of this paper is to present and discuss a range of in-situ and laboratory methods can be used to measure the morphological characteristics of porous materials. In the case of foams and granular media used for noise control applications, the most common of these characteristics are pore size and its distribution, porosity, and pore connectivity. In the case of fibrous media, the most useful non-acoustical characteristics are the fibre size and packing density. In the case of outdoor surfaces such as gravel, sandy soils, and agricultural land, which can be partly saturated with water, it is of direct interest to measure the degree of water saturation hydraulic permeability and pore size gradient. In the case of materials with good physiochemical properties, it is of interest to determine their internal pore surface area, porosity, and pore size scales. In this paper, the existing and newly emerging acoustic characterisation methods are discussed in terms of their complexity, accuracy, and sample requirements. The application of these methods relate to traditional needs to measure and predict the in-situ performance of noise control elements and outdoor surfaces, to ensure the quality control during material manufacturing process and to be able to measure non-invasively the micro- and nano-structure of porous media which is used in catalytic filters, electric capacitors and for gas storage. Parts of this paper were presented at the 22nd International Congress on Sound and Vibration in Florence.
\end{abstract}

\section{INTRODUCTION}

Traditionally, models for the acoustical properties of porous media have been developed and applied directly to predict sound propagation in porous rocks (geophysics, seismology, and exploration ${ }^{1,2}$ ), sound propagation in the presence of underwater sediments (underwater acoustics, geophysic ${ }^{3}$ ) and airborne sound propagation in the presence of porous soils (outdoor noise control ${ }^{4}$ ). It appears that the most common use of these models have been used to predict the acoustical properties of materials are manufactured for noise control. ${ }^{5}$ These materials are reticulated foams, fibrous, and granular media are able to absorb a considerable proportion of the energy in the incident sound wave.

However, in an overwhelming majority of practical engineering problems, the acoustical properties of porous materials are of little or no interest, the relationship between the acoustical properties, pore, and frame morphology are of significant interest. In applications related to energy storage, it is important to measure the porosity and tortuosity of ceramic separators, which control the electrolyte uptake by the porous separator and its ability to conduct an electrical current. In applications related to filtration operations, similar characteristics to be measured routinely to determine the permeability of the membrane in the presence of a flow of fluid. In pharmaceutical applications, it is often of interest to measure the mean particle size and compaction, particle size distribution, and the amount of moisture absorbed by the particle mix. In chemistry and chemical engineering applications, it is important to know the internal pore surface area of materials are used to deliver catalysts to control the chemical reaction and convert noxious substances in chemically inert bonds.

Therefore, the purpose of this paper is to illustrate how mod- els of the acoustical properties of porous media of varying complexity can be used to determine the morphological characteristics of the material frame and pores. The paper is focused on the basic physical relations, which can be used in the parameter inversion process whereby a measurable acoustical property can be directly related to the pore/frame morphology. The paper also explains how to measure the right acoustical properties and use them correctly in the parameter inversion process.

\section{METHODS FOR PARAMETER INVERSION BASED ON ASYMPTOTIC BEHAVIOUR}

The main aim of this section is to show how the morphological characteristics of the porous material's frame and pores can be extracted from those acoustical properties of which asymptotic behaviour has a clear physical meaning. The focus will be on rigid-frame porous media, for which the values of the frame density and bulk modulus are considerably greater than that of the filling fluid. This approximation is true in most cases when the material's pores are saturated by a gas. The fluid trapped in these pores is typically presented as a homogeneous, equivalent fluid with complex, frequency dependent characteristic impedance $z_{b}(\omega)$, and complex wavenumber $k_{b}(\omega)$. Where $\omega=2 \pi f$ is the angular frequency, where $f$ is the frequency in Hertz. The value of the characteristic impedance and the boundary conditions surrounding the porous layer determine the ability of sound waves to penetrate this layer. The value of the complex wavenumber relates to the speed of the sound wave in the porous space and the rate at which it attenuates.

The frequency-dependent behaviour of the characteristic impedance and propagation constant can be predicted theoretically or empirically. Theoretical models tend to treat the 
viscous and thermal effects separately by introducing complex expressions for the dynamic, frequency-dependent density $\rho_{b}(\omega)$, and complex compressibility $C_{b}(\omega)$, of the fluid. The viscous friction losses in the oscillatory flow in a porous material are controlled by the non-zero imaginary part of the dynamic density and are pronounced at frequencies below the so-called Biot frequency, $\omega_{v}=\frac{8 \eta}{s^{2} \rho_{0}}$ (see Eq. (11) in ref. ${ }^{9}$ and Eqs. (4.144), (5.3) in ref. ${ }^{5}$ ), where $\eta$ is the dynamic viscosity, $\rho_{0}$ is the equilibrium density of air and $s$ is the characteristic pore size. Above this frequency, the viscous friction is relatively small and the inertial forces are predominant. Therefore, the value of this frequency is of importance since it separates the viscous (low frequency, $\omega \ll \omega_{v}$ ) and inertial (high frequency, $\omega \gg \omega_{v}$ ) flow regimes in the pore which are basically controlled by the characteristic pore size. Similarly, the thermal losses in a porous medium are controlled by the non-zero imaginary part of the complex compressibility and are pronounced around the characteristic thermal frequency, $\omega_{t}=\frac{\kappa}{s^{2} \rho_{0} C_{p}}$, where $\kappa$ is the thermal conductivity of the saturating fluid and $C_{p}$ is the specific heat of fluid at constant pressure. This frequency separates the isothermal (lowfrequency, $\omega \ll \omega_{t}$ ) and adiabatic (high-frequency, $\omega \gg \omega_{t}$ ) regimes. In the case of air-filled fibreglass, which has a mean pore size $s \simeq 100 \mu \mathrm{m}$ at room temperature, the values of the above frequencies are $\omega_{v} \simeq 14480 \mathrm{rad} / \mathrm{s}\left(f_{v} \simeq 2300 \mathrm{~Hz}\right)$ and $\omega_{t} \simeq 2150 \mathrm{rad} / \mathrm{s}\left(f_{t} \simeq 342 \mathrm{~Hz}\right)$.

The complex characteristic impedance and and wavenumber are linked to the dynamic density and complex compressibility by the following expressions:

$$
z_{b}(\omega)=\sqrt{\rho_{b}(\omega) / C_{b}(\omega)} \text { and } k_{b}=\omega \sqrt{\rho_{b}(\omega) C_{b}(\omega)} .
$$

Unlike theoretical models, empirical models do not generally bother with deriving $\rho_{b}(\omega)$ and $C_{b}(\omega)$ and estimate directly the characteristic impedance, $z_{b}(\omega)$, and complex wavenumber, $k_{b}(\omega) .{ }^{8,10}$ The expressions in these models are typically based on statistically significant experimental data to these models can closely fit. A common acoustical property that is directly measurable is the normal incidence surface impedance of a hard-backed porous layer of thickness $d(i=\sqrt{-1})$ :

$$
z_{s}(\omega)=z_{b}(\omega) \operatorname{coth}\left(-i k_{b}(\omega) d\right) .
$$

It is clear that this property depends both on the characteristic impedance of the porous material and on its wavenumber. Figure 1 shows the experimental setup for measuring the surface impedance using the standard two-microphone impedance tube method. ${ }^{11}$ The equipment for this experiment is available from a number of manufacturers, e.g., from Bruel and Kjaer (http://bksv.com/), Mecanum Inc. (http://www.mecanum. com/impedance-tube.html), Materiacustica srl (http://www. materiacustica.it/mat_UKProdotti_3Mics.html), or AFD mbH http://www.akustikforschung.de. The acoustic absorption coefficient spectrum is often used as an estimate of the proportion of the incident sound energy a porous layer is able to absorb. The normal incident absorption coefficient is calculated as

$$
\alpha(\omega)=1-\left|\frac{z_{s}(\omega)-\rho_{0} c_{0}}{z_{s}(\omega)+\rho_{0} c_{0}}\right| ;
$$

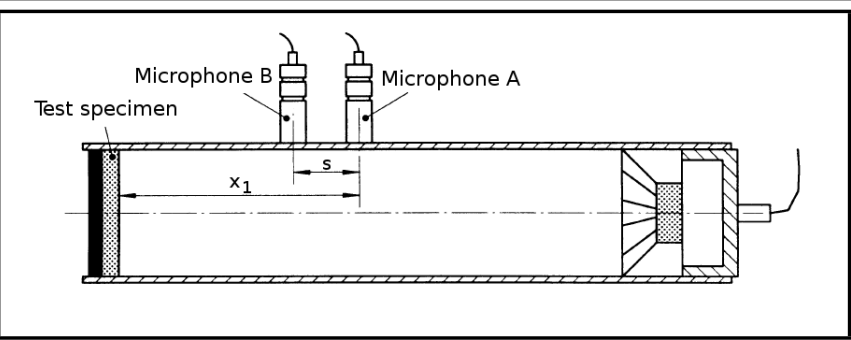

Figure 1. 2-microphone impedance tube setup to measure the surface impedance of a porous layer. ${ }^{11}$

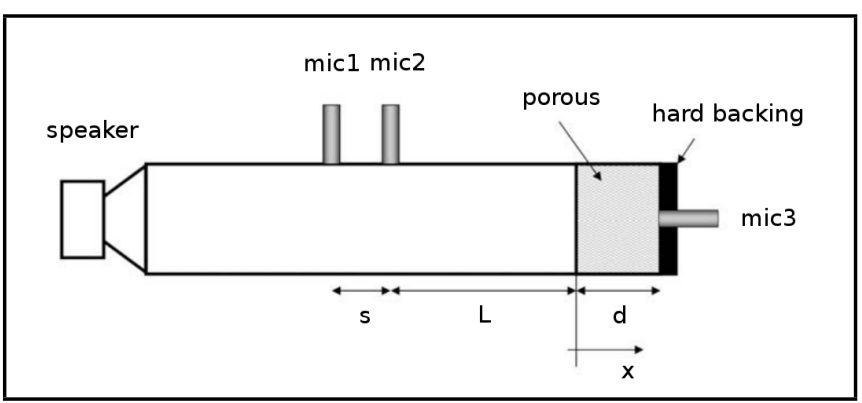

Figure 2. A 3-microphone impedance tube setup to measure the surface impedance of a porous layer. ${ }^{16}$

where $c_{0}$ is the sound speed in fluid. The reproducibility of this method is discussed in ref., ${ }^{12}$ where it was shown that the variation in the measured acoustical properties can be considerable if the material is not homogeneous. In the case of materials with high flow resistivity, the accuracy of this method is also dependent on the quality of the sample preparation and on the presence of the circumferential gap between the material sample and the wall of the impedance tube as shown in references. ${ }^{13,14}$ The flow resistivity, elastic properties of the material frame, and its density are the main parameters which can alter the behaviour of the material specimen constrained in the impedance tube. ${ }^{14}$ In this respect, it is common to measure the acoustical properties of at least three material specimens and to work with their average values, as it is done in the examples presented in this paper.

Obviously, the acoustic surface impedance $z_{s}(\omega)$, is not a fundamental acoustical property because it depends on the material geometry and boundary conditions. An alternative laboratory method to measure more fundamental acoustical properties of porous media is to use the three-microphone impedance tube as suggested originally by Iwase ${ }^{15}$ and subsequently revisited by Doutres, et al. ${ }^{16}$ Figure 2 illustrates the basic setup that can be used to measure directly the characteristic impedance and complex wavenumber. These properties can be used to estimate the dynamic density and complex compressibility from the following equations:

$$
\rho_{b}(\omega)=z_{b}(\omega) k(\omega) / \omega \text { and } C_{b}(\omega)=k(\omega) /\left(z_{b}(\omega) \omega\right) .
$$

As an example, equipment for three-microphone impedance tube experiment is available from Materiacustica (http://www.materiacustica.it). Alternatively, it is possible to extract the characteristic impedance and complex wavenumber from the two-microphone impedance tube measurements using either the "two thickness" or "two cavity" methods as described in reference. ${ }^{17}$ 


\subsection{Reticulated Foams and Fibrous Materials}

Light-weight, fibrous materials (e.g., fibreglass, rockwool, and glassfibre) and reticulated foams (e.g., polyurethane and melamine open cell foams) are the simplest case of porous media for which the values of porosity (see Eq. (2.25) in ref. ${ }^{5}$ for a formal definition of porosity) and tortuosity (see Eq. (5.23) in ref. ${ }^{5}$ for a formal definition of tortuosity) are very close to unity and their pore size is relatively constant. For these materials the only meaningful parameter which controls the behaviour of the characteristic impedance and wavenumber is the flow resisitivity, $\sigma$ (see Eq. (2.27) in ref. ${ }^{5}$ for the formal definition of flow resistivity). For these porous media, the porosity can be set to unity or if needed, measured directly by using a number of non-acoustic methods, e.g., by comparison of air volume method ${ }^{18}$ or by measuring the sample density and referring it to the frame density.

Since this is for an overwhelming majority of reticulated foams $\alpha_{\infty}, \phi \simeq 1$ (see the work of Bonfiglio and Pompoli on polyurethane foams for which $\alpha_{\infty}>1^{19}$ ), it can be shown that the low frequency limit $\left(\omega \ll \omega_{v}\right)$ for the dynamic density is dependent on the flow resistivity alone, i.e.,

$$
\rho_{b}(\omega) \simeq \frac{\alpha_{\infty} \rho_{0}}{\phi}\left(1+\beta-\frac{\sigma \phi}{i \omega \rho_{0} \alpha_{\infty}}\right) .
$$

The parameter

$$
\beta=\frac{4}{3} e^{4\left(\sigma_{s} \ln 2\right)^{2}} ;
$$

in the above equation is the Pride parameter ${ }^{9}$ discussed later at the end of this section making reference to the work by Horoshenkov et al. ${ }^{20}$ The above expression suggests that the flow resistivity can be determined from the asymptotic behaviour of the imaginary part of the dynamic density by estimating the low-frequency limit $\left(\omega \ll \omega_{v}\right)$ of

$$
\sigma \simeq-\operatorname{Im}\left(\rho_{b}(\omega) \omega\right)
$$

Figure 3 shows the behaviour of Eq. (7) plotted as a function of frequency for reticulated foam (see Fig. 4) with $\sigma=9470$ $\mathrm{Pa} \mathrm{s} \mathrm{m} \mathrm{m}^{-2}, \phi=0.910$, and $\alpha_{\infty} \simeq 1.00$. As the frequency of sound reduces considerably below the viscous characteristic frequency $f_{v}$, the value of $-\operatorname{Im}\left(\rho_{b}(\omega) \omega\right)$ reduces to $\sigma$ allowing for the estimation of the flow resistivity from the acoustical data.

In many cases, it is of interest to estimate the material's pore size from the flow resistivity data. This procedure requires us to make an assumption for the shape of material pores. The choice of pore geometry for porous media with $\phi$ and $\alpha_{\infty}$ being close to unity is not critical in this procedure. However, the choice of the characteristic pore dimension for the adopted pore geometry is important. In the case of a circular cylindrical pore, the dimension is the pore radius and in the case of triangular pore shape it is the side of the triangle. In the case of a slit, the characteristic dimension is the half-width of the slit. In the case of any other arbitrary pore geometry the procedure for choosing of the right pore dimension can be completed through the estimation of the pore's hydraulic radius and assignment of the right shape factors as explained. ${ }^{6}$

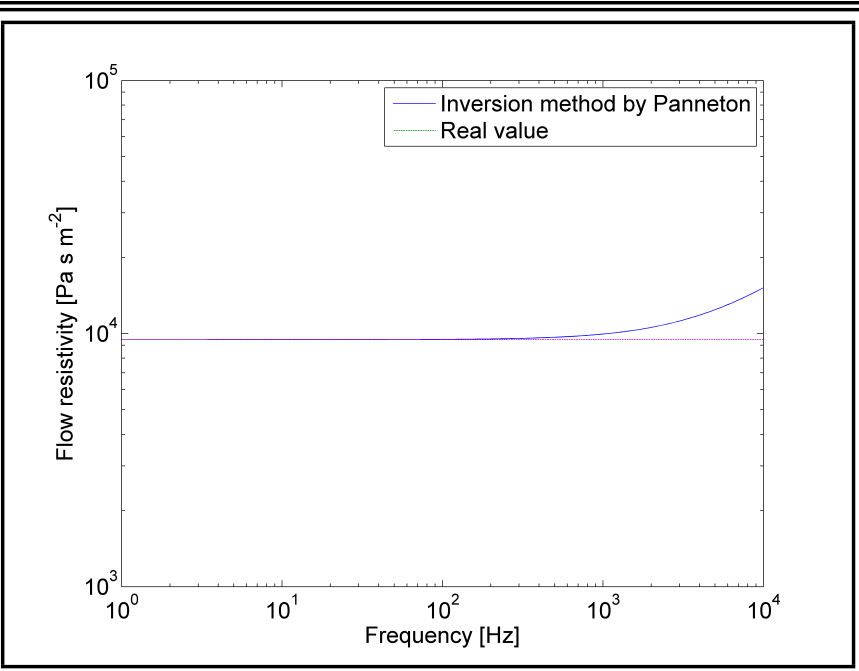

Figure 3. The behaviour of the function $-\operatorname{Im}\left(\rho_{b}(\omega) \omega\right)$ showing its asymptotic convergence to the value of the static air flow resistivity of the melamine foam sample as $\omega \rightarrow 0$.

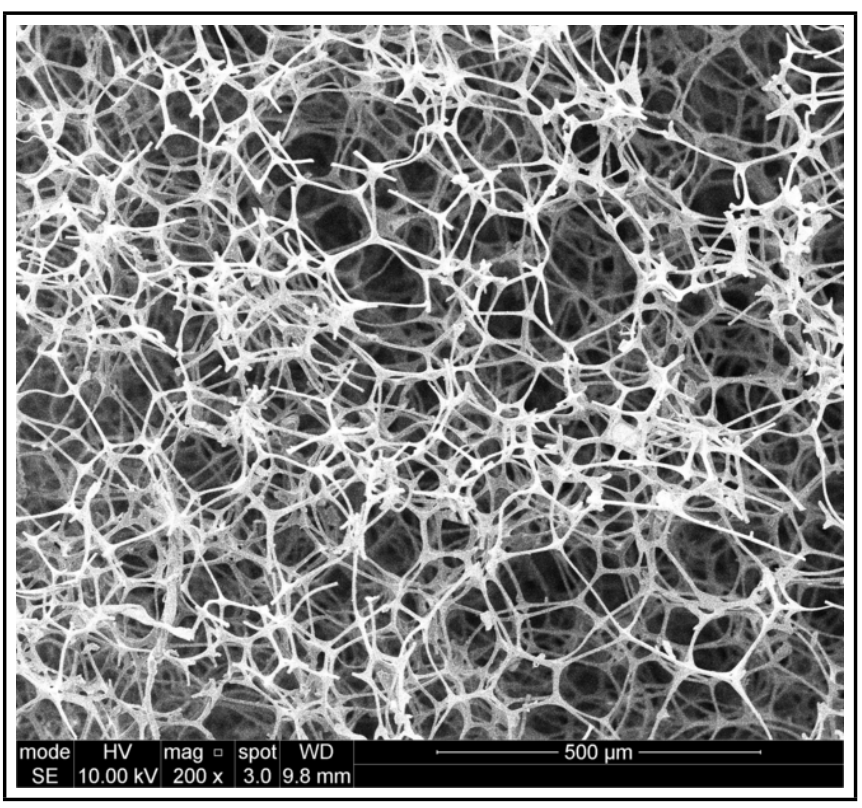

Figure 4. A microscopic photograph of reticulated foam which pore size was estimated from acoustical data.

Going back to the previously considered example with reticulated foam, we can assume that the pore geometry is circular and that the pore size is relatively uniform. In this case the pore radius $s$ can be estimated from the following equation (see Eq. (51) in ref. ${ }^{6}$ )

$$
s=\sqrt{\frac{8 \eta}{\sigma \phi}}
$$

For the reticulated foam sample considered here $(\sigma=9470 \mathrm{~Pa}$ $\mathrm{s} \mathrm{m}^{-2}, \phi=0.910$ and $\eta=1.81 \times 10^{-5} \mathrm{~Pa} \mathrm{~s}$ ) Eq. (8) yields $s=129 \mu \mathrm{m}$, which corresponds well to the pore dimension typical to melamine foams ${ }^{21}$ and $s=128 \pm 67 \mu \mathrm{m}$ estimated from the optical image analysis performed independently on this particular foam sample using ImageJ software. ${ }^{7}$ The value of porosity is slightly lower than that expected for reticulated foam. This can be explained by the fact that not all the pores in this material are interconnected and a proportion of the pores are closed. 


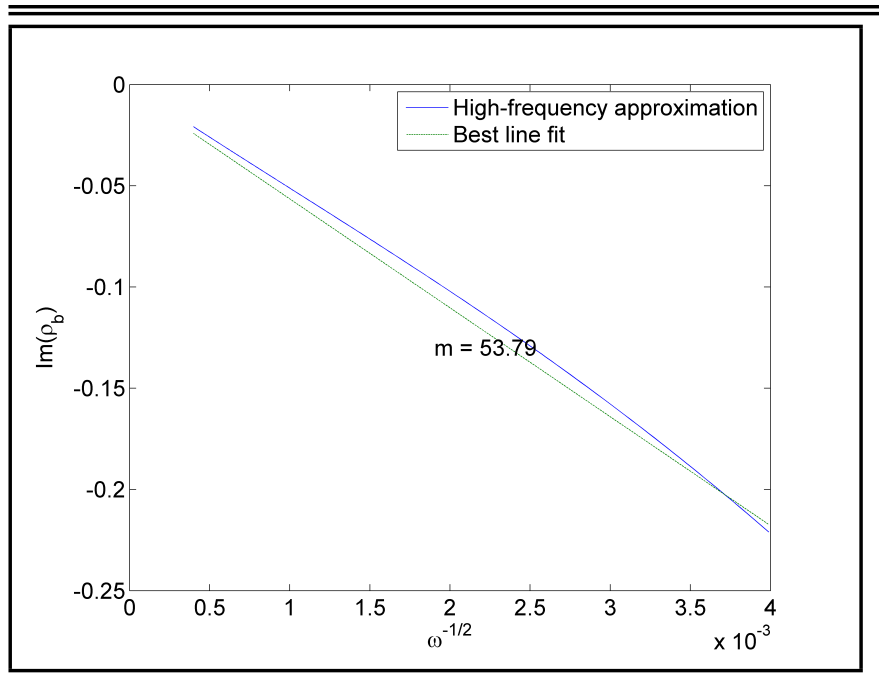

Figure 5. The behaviour of approximation (10) (solid line) and best line fit to this approximation (dashed line) from which the mean slope $m$ can be determined and used to estimate the characteristic pore size of reticulated form (see Fig. 4) with Eq. (11).

An alternative way to estimate pore size from the acoustical data is to use the high-frequency asymptotic limit for the dynamic density as proposed by Bonfiglio and Pompoli. ${ }^{22}$ This approach is based on the behaviour of high-frequency asymptotic limit $\left(\omega \gg \omega_{v}\right)$ for the imaginary part of the dynamic density, which is described by

$$
\rho_{b}(\omega) \simeq \frac{\alpha_{\infty}}{\phi} \rho_{0}\left(1+\frac{2}{s} \sqrt{\frac{\eta}{-i \omega \rho_{0}}}\right) .
$$

This equation was adapted to describe the high-frequency asymptotic behaviour of the dynamic density of a bulk porous medium by dividing the original equation by Johnson et al. (see Eq. (2.16a) in ref. ${ }^{23}$ ) by the porosity $\phi$. With respect to $\omega^{-1 / 2}$, the high-frequency behaviour of the imaginary part of (9) is

$$
\operatorname{Im}\left(\rho_{b}(\omega)\right) \simeq-m \omega^{-1 / 2} ;
$$

where $m=\frac{\alpha_{\infty} \rho_{0}}{s \phi} \sqrt{\frac{2 \eta}{\rho_{0}}}$ is the slope of the asymptote. In this case, the characteristic pore dimension can be estimated from

$$
s=\frac{\alpha_{\infty} \rho_{0}}{m \phi} \sqrt{\frac{2 \eta}{\omega \rho_{0}}} .
$$

Figure 5 shows the high-frequency asymptotic behaviour of the imaginary part of the dynamic density (Eq. (10) plotted against $\omega^{-1 / 2}$ (solid line)). This function was calculated for the reticulated foam sample in the frequency range of $10-1000 \mathrm{kHz}$, which was chosen well above the viscous characteristic frequency for this material $\left(f_{v}=1.1 \mathrm{kHz}\right)$ to ensure the validity of this high-frequency approximation. Figure 5 also presents the expression of the best linear fit (dashed line), which has a slope of $m=53.79$. At ambient temperature $\phi=0.91$ and $\alpha_{\infty}=1.00$, the substitution of this slope in Eq. (11) yields the characteristic pore dimension of $132 \mu \mathrm{m}$, which is within $2 \%$ of the value estimated from the flow resistivity data.

A similar approach for microstructure characterisation can be applied in the case of fibrous materials, e.g., polyester fibre. However, in the case of fibre, it is more important to estimate the fibre diameter and material density. These parameters can be estimated from the flow resistivity value, which

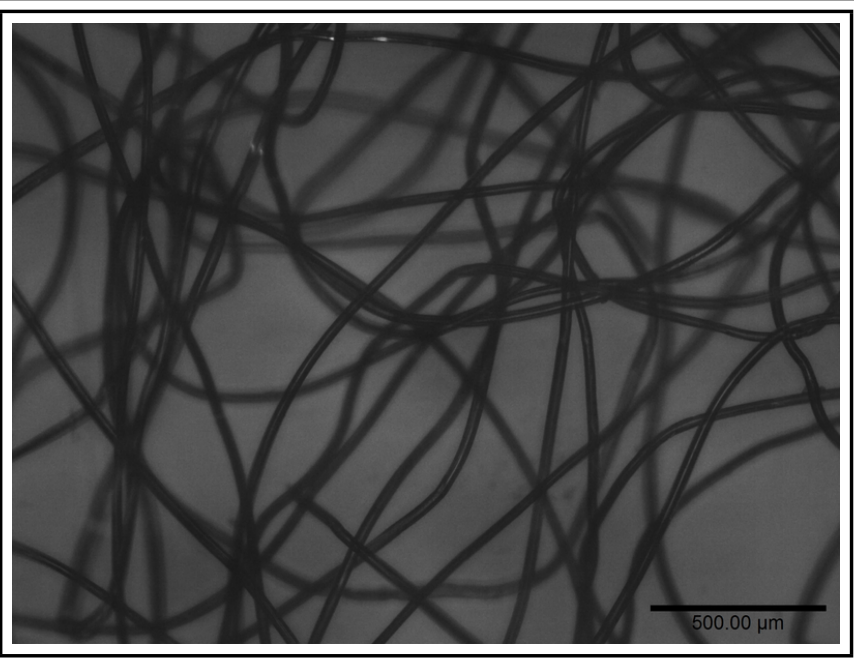

Figure 6. The diameter of this polyester fibre (photographed) was estimated from acoustical data.

can be measured acoustically as described in the previous paragraphs. There are several models that can be used to link the fibre diameter $d_{f}$, and material density $\rho_{f}$, with the flow resistivity, $\sigma{ }^{24,25}$ However, a better model to enable this link is the Carman-Kozeny model for the permeability of porous media, ${ }^{26}$ which was introduced in the 1930s. Unlike some other empirical models, which can be used to predict the flow resistivity of fibre, the Carman-Kozen model is derived from Poiseuille's equation for laminar flow and presents a theoretical links between the flow resistivity, fibre diameter, and bulk material density. The model suggests the following relationship between the static flow resistivity, porosity and fibre diameter:

$$
\sigma=\frac{180 \eta(1-\phi)^{2}}{d_{f}^{2} \phi^{3}} .
$$

Because the density $\rho_{f}$, of the fibre's material and material sample density $\rho_{m}$, are usually known (e.g., in the case of the polyester fibre $\rho_{f}=1380 \mathrm{~kg} / \mathrm{m}^{3}$ and the sample's density is a directly measurable quantity), then the porosity is also easily calculated from $\phi=1-\rho_{m} / \rho_{f}$. Assuming that the tortuosity of this media is close to unity, the only parameter which is left to invert from the flow resistivity data is the fibre diameter. It can be estimated as

$$
d_{f}=\sqrt{\frac{180 \eta(1-\phi)^{2}}{\sigma \phi^{3}}} .
$$

The application of Eq. (13) to $30 \mathrm{~kg} / \mathrm{m}^{3}$ Den 15 polyester fibre (see Fig. 6) suggests the value of $d_{f}=35.6 \mu \mathrm{m}$. This estimate is obtained for $\sigma=1300 \mathrm{~Pa} \mathrm{~s} \mathrm{~m}^{-2}$, which was measured acoustically using the asymptotic limit Eq. (7) and it compares within $10 \%$ with the directly measured 15 Den fibre diameter of $39.2 \mu \mathrm{m}$. In this analysis we assumed that $\phi \sim 1$. It is easy to illustrate that a very similar procedure can be applied to glasswood, rockwool, and fibreglass for which $\phi, \alpha_{\infty} \rightarrow 1$.

\subsection{Granular Materials}

Let us now consider materials with a relatively uniform porous structures which are composed of loose or consolidated 
grains. These materials are characterised by a porosity that is typically in the range of $20-60 \%$, a value that is noticeably less than unity. Accurate modelling of the acoustical properties of these materials cannot be based on flow resistivity data alone. The value of the tortuosity for these media is larger than unity and it is important to include this in the model together with the true value of the porosity to describe accurately the behaviour of the acoustic characteristic impedance and the complex wavenumber in the medium to high frequency range. Therefore, modelling of the acoustical properties of granular materials effectively requires the knowledge of the following three parameters: flow resistivity, $\sigma$, porosity, $\phi$, and tortuosity $\alpha_{\infty}$

The physical meaning of the flow resistivity and porosity is generally well understood. This is not often the case with the tortusity and it is appropriate to say a few words about its physical meaning. The existing literature sometimes makes reference to two types of tortuosity: the high-frequency tortuosity limit $\alpha_{\infty}$ (e.g., Eq. (5.21) in ref. ${ }^{5}$ ) and the low-frequency (static) tortuosity limit $\alpha_{0}$ (e.g., section 5.3.6 in ref. ${ }^{5}$ ). Let us focus on $\alpha_{\infty}$, which, in my view is a more useful parameter. There are several definitions for $\alpha_{\infty}$ and some examples include: definition via the structure factor (see Fig. 16 in ref. ${ }^{27}$ ); definition via the high-frequency limit of the dynamic permeability (see Eqs. (2.1c), (2.6b), and (2.9) in ref. ${ }^{23}$ ) and definition using the electrical resistance analogy (see Eq. (20) in ref. ${ }^{28}$ ).

The correct way to define the tortuosity $\alpha_{\infty}$ is to treat it as an apparent reduction in the high-frequency limit for the speed of sound in a porous sample. This apparent reduction is associated with the increase in the length of the path that the sound wave needs to propagate through the tortuous pores in a porous layer rather than propagate in the straight manner in the free space in gas. Mathematically, it can be shown that the high-frequency $\left(\omega \gg \omega_{v}, \omega_{t}\right)$ limits for the dynamic density and complex compressibility (see Eqs. (23) to (25) and (58) in ref. $\left.{ }^{6}\right)$ are $\rho_{b} \simeq \rho_{0} \alpha_{\infty} / \phi$ and $C_{p} \simeq \phi /\left(\gamma P_{0}\right)$ with $P_{0}$ being the ambient atmospheric pressure of gas and $\gamma$ being the ratio of specific heats, respectively. Since $k_{b}(\omega)=\omega \sqrt{\rho_{b} C_{b}}$ and $k_{b}(\omega)=\omega / c_{b}(\omega)$, the above implies that the high-frequency limit for the wavenumber is

$$
k_{b}(\omega) \simeq \omega \sqrt{\alpha_{\infty} \rho_{0} /\left(\gamma P_{0}\right)} ;
$$

i.e., the high-frequency limit of the sound speed in the porous medium $c_{b}(\omega)$, should converge to

$$
c_{b} \simeq c_{0} / \sqrt{\alpha_{\infty}}
$$

where $c_{0}=\sqrt{\gamma P_{0} / \rho_{0}}$ is the adiabatic speed in gas. Obviously, because $\alpha_{\infty}>1$, the sound speed in granular media is always slower than the speed of sound in the filling gas.

Berryman suggested (see Eq. (18) in ref. ${ }^{29}$ ) that the tortuosity of a stack of identical, spherical solid particles can be estimated from the porosity data alone, i.e.,

$$
\alpha_{\infty}=1+\frac{1-\phi}{2 \phi} .
$$

Using acoustical data, the tortuosity can be estimated from the high-frequency behaviour of the squared refraction index

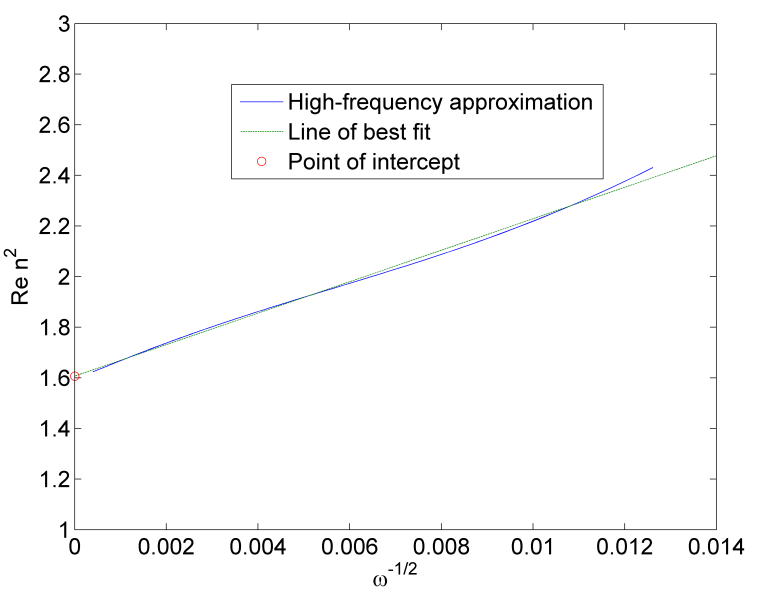

Figure 7. The high-frequency behaviour of real part of the squared refraction index (solid line) and the line of best fit to this behaviour for a stack of $1 \mathrm{~mm}$ diameter glass beads. The red circle illustrates the point of intercept which corresponds with the value of $\alpha_{\infty}$.

$n^{2}(\omega)=\left(c_{0} / c_{p}(\omega)\right)^{2}=\left(k_{b}(\omega) / k_{0}\right)^{2}$, where $k_{0}=\omega / c_{0}$, which converges to $\alpha_{\infty}$ as $\omega \rightarrow \infty$ if $n^{2}(\omega)$ is plotted against $\omega^{-1 / 2}$.

The procedure for the inversion of the tortuosity from refraction index data is well described in detail in ref. ${ }^{22}$ (Eq. (6) in $^{30}$ defines properly the high-frequency asymptotic limit for $k_{b}(\omega)$ ). Because very high frequency data for the refraction index are usually unattainable, then the intercept of the function $n^{2}\left(\omega^{-1 / 2}\right)$ with the ordinate axis is found through a linear fitting procedure as illustrated in Fig. 7 for a stack of 1 $\mathrm{mm}$ diameter glass beads. The acoustically estimated value of the tortuosity for this material is $\alpha_{\infty}=1.61$. This value is within $3 \%$ of the tortuosity estimated from Berryman's formula (Eq. (16)), which yields $\alpha_{\infty}=1.66$. The porosity value $\phi=0.42$, which is used in Berryman's formula was estimated from the material density data, $\phi=1-\rho_{m} / \rho_{g}$ in which the glass density was taken as $\rho_{g}=2500 \mathrm{~kg} / \mathrm{m}^{3}$.

Knowledge of the tortuosity is very helpful, because it enables us to estimate a number of useful non-acoustical parameters of a granular mix. The most obvious parameter is the radius of the granules $R$. The most straightforward way to estimate the radius of the granules is to use Eqs. (13) and (17) in ref. ${ }^{31}$ and the value of the geometrical factor $M=1$ which corresponds to materials with cylindrical pores. ${ }^{23}$ This provides us with the following relationships between the flow resistivity, porosity and the radius of granules

$$
R=\frac{9(1-\phi)}{4(1-\Theta) \phi \alpha_{\infty}} s
$$

where the characteristic pore dimension

$$
s=\sqrt{\frac{8 \eta \alpha_{\infty}}{\phi \sigma}} .
$$

and $\Theta=3 /(\pi \sqrt{2})(1-\phi)$ (see Eq. (19) in ref. ${ }^{31}$ ). The parameter $s$ in Eq. (18) is effectively the pore radius, which for materials with assumed cylindrical pores, is equivalent to the characteristic viscous length, $\Lambda \equiv s$ (see Eq. (2.17) in ref. ${ }^{23}$ for the formal definition of $\Lambda$ ). Acoustical data obtained for 


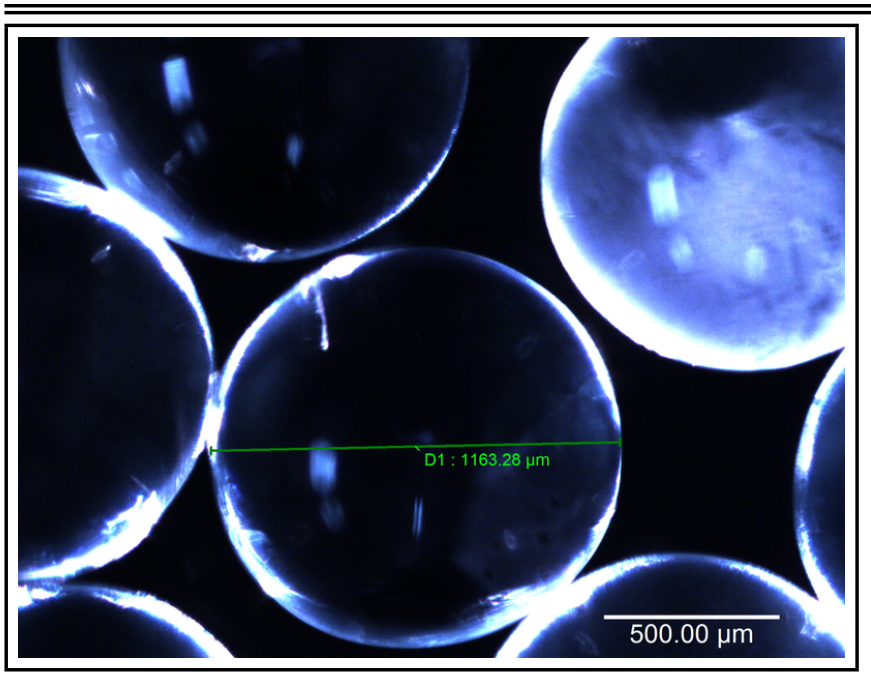

Figure 8. A microscopic photograph of glass beads which diameter was estimated from acoustical data.

a stack of $1 \mathrm{~mm}$ diameter glass beads, Eq. (18) together with the flow resistivity and porosity data enables us to estimate the characteristic pore dimension (length) for this granular material, which is $s=173 \mu \mathrm{m}$. Here, we used the flow resistivity value of $\sigma=18.4 \mathrm{kPa} \mathrm{s} \mathrm{m}^{-2}$, which was estimated from the low-frequency limit of the imaginary part of the dynamic density (see Eqs. (5) and (7)). If we substitute this value of $s$ in Eq. (17), then the radius of the glass beads in the stack can be estimated as $R=532 \mu \mathrm{m}$, which can then be within $9 \%$ of the true radius of these glass beads, which is estimated from the optical images to be $582 \mu \mathrm{m}$ (see Fig. 8).

Another use of the characteristic pore dimension determined acoustically is the estimate of the specific pore surface area $S_{s}=S / V$, where $V$ is the volume of pores in $\mathrm{m}^{3}$ and $S$ is the pore surface area in $\mathrm{m}^{2}$. This information is important in chemical engineering, energy storage, and pharmaceutical powder manufacturing. In our example, the theoretical value for the specific pore surface area of a stack of perfectly round grains is easy to estimate analytically as $S_{s}=3 / R$, which, in the case of the glass beads with $R=582 \mu \mathrm{m}$, is $S_{s}=5.15 \times 10^{3} 1 / \mathrm{m}$. If we use the value of $R=532 \mu \mathrm{m}$ determined acoustically, then the specific pore surface area is estimated as $S_{s}=5.64 \times 10^{3} 1 / \mathrm{m}$, which is $9 \%$ greater than the theoretical value of $S_{s}$.

I would like to make a comment on the definition and use of low-frequency (static) tortuosity $\alpha_{0}$, which may be of importance in the case of materials for which $\phi<1$ and $\alpha_{\infty}>1$. In ref. ${ }^{5}$ this quantity is defined as the low-frequency limit of the dynamic tortuosity function, which is effectively $\alpha(\omega)=$ $\phi \rho_{b}(\omega) / \rho_{0}$. Pride et al. ${ }^{9}$ showed that the low-frequency limit of this function is given by Eq. (5). In this equation the parameter $\beta$ is the so-called Pride parameter. In some more recent publications this parameter has been modified and assigned with notations other than $\beta$ which was originally used by Pride et al. (e.g., parameter $P$ in Eq. (25) in ref., ${ }^{32}$ parameter $b$ in Eq. (5.32) in ref. ${ }^{5}$ ). Although limited attempts have been made to provide some physical interpretation of this parameter, its physical meaning can appear ambiguous and makes sense to show how it is related to the low-frequency (static) tortuosity, pore geometry and pore size.
From the above definition of $\alpha_{0}$ (also see section 5.3.6 in ref. ${ }^{5}$ for some limited discussion on this issue) and from the low-frequency limit of the dynamic density (see Eq. (5)), it seems obvious that $\alpha(\omega) \simeq \alpha_{\infty}(1+\beta)$ as $\omega \rightarrow 0$. In ref. ${ }^{20}$ it was shown that in the case of a porous medium with cylindrical pores which radius is log-normally disctributed; the Pride parameter is equivalent to $\beta=4 / 3 e^{4\left(\sigma_{s} \ln 2\right)^{2}}-1$, where $\sigma_{s}$ is the standard deviation in pore size distribution estimated using a logarithmic scale with the base of 2 . This implies that the low-frequency (static) tortuosity can be estimated as

$$
\alpha_{0}=\frac{4}{3} \alpha_{\infty} e^{4\left(\sigma_{s} \ln 2\right)^{2}}
$$

which is consistent with the value of $\alpha_{0}=\frac{4}{3} \alpha_{\infty}$ derived for a medium with identical, cylindrical pores, i.e., when $\sigma_{s}=0$. It can be noted that the low-frequency (static) tortuosity is always greater than its high-frequency counterpart, i.e., $\alpha_{0}>\alpha_{\infty}$. It can also be noted that the value of the coefficient $4 / 3$ in Eq. (19) is related to the choice of the pore geometry (4/3 is for circular pores) and it is determined by the ratio of the pore shape factors (see the ratio of pore shape factors $\bar{\psi}_{1} / \bar{\psi}_{0}^{2}$ in Table II in ref. ${ }^{6}$ ). In the case of pores of triangular geometry, this factor becomes $10 / 7$. In the case of slit-like pores, it is $6 / 5$. In the case of a medium with arbitrary pore shape, it needs to be estimated numerically. ${ }^{32}$ The value of $\alpha_{0}$ increases with the increased value of the standard deviation in the pore size $\sigma_{s}$, and doubles when $\sigma_{s}$ increases from 0 (uniform, identical pores, e.g., glass beads) to 0.6 (pronounced pore size distribution, e.g., recycled granulates of a highly irregular shape).

\subsection{Sandy Soils}

A common type of porous media are sandy soils. The acoustical properties of these materials have been studied extensively, mainly with respect to outdoor sound propagation and noise control. ${ }^{4}$ It is often of interest to use sound to measure remotely the permeability of soils and their moisture content. Applications of the acoustic parameter inversion technology can be of use in agriculture and geotechnics.

The porosity of a majority of outdoor soils is low, whereas their flow resistivity and tortuosity are high. A usual criteria for soil to be of high flow resistivity is the ratio $\omega / \sigma \ll 1$. A layer of soil is typically thick in comparison with the acoustical wavelength. This combination of properties enables the application of relatively simple engineering models, which are based on no more than two non-acoustical parameters and which assume that the layer thickness is semi-infinite. These models are easy to use in the inversion process from which the non-acoustical data can be determined from the acoustical impedance. The acoustical impedance of these media can be measured in-situ in a number of ways. ${ }^{33-35}$ In the laboratory, their acoustical properties can be measured in an impedance tube, which can be adapted to simulate the effect of moisture. ${ }^{36}$

A good model for the acoustical properties of these materials is the 2-parameter Attenborough model (model NHBL2A, Eq. (12) in ref. ${ }^{37}$ ). It is not easy to follow the discussion presented $\mathrm{in}^{37}$ and it makes sense to give the expression for this 
model here explicitly

$$
z_{s}(f)=0.436 \sqrt{\sigma_{e} / f}-i\left(0.436 \sqrt{\sigma_{e} / f}+9.75 \alpha_{e} / f\right)
$$

where $\sigma_{e}$ is the effective flow resistivity of soil $\alpha_{e}$ is the rate of porosity change and $f$ is the frequency of sound in Hertz. The effective flow resistivity in the above model can be adjusted to match the measured data for the real part of the surface acoustic impedance. The imaginary part of the acoustic impedance the effective rate of change in porosity can also be subsequently adjusted to match the imaginary part of the surface impedance measured at the frequency points $f_{n}$. The application of the least squares method yields ${ }^{36}$

$$
\log \sigma_{e}=-2 \log \left[\frac{0.436 \sum_{n} f_{n}^{-1}}{\sum_{n} \operatorname{Re}\left(z_{s}\left(f_{n}\right)\right) / \sqrt{f_{n}}}\right]
$$

and

$$
\log \alpha_{e}=-\frac{\sum_{n} \operatorname{Im}\left(z_{s}\left(f_{n}\right)\right) / f_{n}+0.436 \sqrt{\sigma_{e}} \sum_{n} f_{n}^{-1.5}}{9.48 \sum_{n} f_{n}^{-2}} .
$$

Figure 9 shows the spectra of the measured acoustic surface impedance and impedance fitted using Eqs. (21) and (22) for a $50 \mathrm{~mm}$ thick layer of fine sand with variable degree of moisture (see the parameter $S$ above each of the four graphs). The predictions were obtained using the following inverted values of the effective flow resistivity and rate of porosity change: $S=0 \%, \sigma_{e}=146 \mathrm{kPa} \mathrm{s} \mathrm{m}^{-2}$ (i.e., $\omega / \sigma_{e} \ll 1$ stands for the frequency range considered here), $\alpha_{e}=-27.71 / \mathrm{m}, S=15 \%$, $\sigma_{e}=144 \mathrm{kPa} \mathrm{s} \mathrm{m}^{-2}, \alpha_{e}=1321 / \mathrm{m}, S=48 \%, \sigma_{e}=151 \mathrm{kPa}$ $\mathrm{s} \mathrm{m}^{-2}, \alpha_{e}=6231 / \mathrm{m}$; and $S=95 \%, \sigma_{e}=40.9 \mathrm{kPa} \mathrm{s} \mathrm{m}^{-2}$, $\alpha_{e}=11261 / \mathrm{m}$. The inverted value of the rate of porosity change $\alpha_{e}$ almost linearly relates to the moisture content $S$, which enables us to determine it remotely from the acoustical data. The effective flow resistivity of the dry sand $(S=0)$ can be converted to the permeability of sand

$$
\kappa_{p}=\eta / \sigma_{e} ;
$$

whereas the predicted/measured impedance can be used to estimate the uniformity coefficient $C_{u} \cdot{ }^{38}$ This is of importance in soil mechanics and geotechnics.

\section{OPTIMISATION METHODS FOR PARAMETER INVERSION}

Details of some analytical procedures for parameter inversion for materials that acoustical properties need to be described by relatively complex models can be found in refs. ${ }^{39,40}$ These procedures require accurate data on the dynamic density $\rho_{b}(\omega)$ and complex compressibility $C_{b}(\omega)$, which are not often easy to measure. As a result, a number of other inversion methods have been proposed to determine the non-acoustical parameters of complex porous media from acoustical data for the absorption coefficient and surface impedance spectra. A good summary of these methods can be found in Chapter 4 in the work by Bonfiglio. ${ }^{41}$ These methods do not require the relatively complicated procedures and equipment needed to measure the characteristic impedance and complex wavenumber.
It makes sense to apply these methods to materials with a very simple and relatively uniform pore structure which can be described by one parameter only (e.g., reticulated foams), or to materials which have a very complex pore structure and requires a model with several parameters (e.g., complex granular media).

The most straightforward approach to this type of parameter inversion is to adopt a suitable optimisation method and prediction model to minimise the difference

$$
F(\mathbf{x})=\sum\left|g_{\exp }\left(\omega_{n}\right)-g_{t h}\left(\omega_{n}, \mathbf{x}\right)\right| ;
$$

where $g_{\exp }\left(\omega_{n}\right)$ is the measured absorption coefficient or surface impedance spectrum and $g_{t h}\left(\omega_{n}\right)$ is its modelled counterpart. The vector $\mathbf{x}$ here is the design variable vector which is composed of the parameters with unknown values and need to be determined with the help of minimisation procedure Eq. (24). The sum in Eq. (24) is taken over all the frequency points $\omega_{n}$ for which the measured values of the function $g_{\text {exp }}\left(\omega_{n}\right)$ are available. The choice of the model here is important because the more complex the model is, the more parameters it will be required to fit in this model. If more parameters will be required to fit, then the rank of the design vector will be higher, and there will be more ambiguity in the inverted parameter values because the function $F(\mathbf{x})$ is usually multivalued. In order to reduce the ambiguity, it is common to use a constrained optimisation method ${ }^{42}$ and the values of porosity and flow resistivity which can be measured using the non-acoustical procedures..$^{18,43}$

\subsection{Reticulated Foams}

Let us illustrate this parameter inversion process for a few different types of porous media with increasing degree of complexity. As before, we begin with the simplest form of porous media, which are reticulated foams and fibrous materials. Modelling the acoustical behaviour of these materials does not require a complicated model because it is dominated by the flow resistivity alone as it is mentioned already in section 2.1. Therefore, it is a relatively straightforward way to apply a direct optimisation procedure to adjust the flow resistivity value to match the measured data and predictions. Figure 10 shows the match between the measured and fitted real and imaginary parts of the impedance of a $17.5 \mathrm{~mm}$ thick, hardbacked layer of melamine foam. This fitting was achieved through the described minimisaton procedure in which the function $g\left(\omega_{n}\right)=z_{s}\left(\omega_{n}\right), z_{s}\left(\omega_{n}\right)$ being the normal incidence surface impedance (see Eq. (2)) measured at 660 frequency points in the range between 200 and $4000 \mathrm{~Hz}$ using the 2microphone method in $45 \mathrm{~mm}$ diameter impedance tube. Here we used a very simple, constrained Nelder-Mead optimisation algorithm ${ }^{44}$ in which we let $\mathbf{x}=\sigma$ to be the design variable, while the porosity and tortuosity were ignored by setting them to unity, i.e. $\phi=1$ and $\alpha_{\infty}=1$. The model used in this example was the Páde approximation model ${ }^{61}$ which depends on the four directly measurable parameters: porosity, flow resistivity, tortuosity and standard deviation in the log-normally

\footnotetext{
${ }^{1}$ References ${ }^{45,46}$ may be more useful here because they present applications of the Páde approximation model to real life porous media and include parameter inversion applications (ref. $\left.{ }^{46}\right)$.
} 


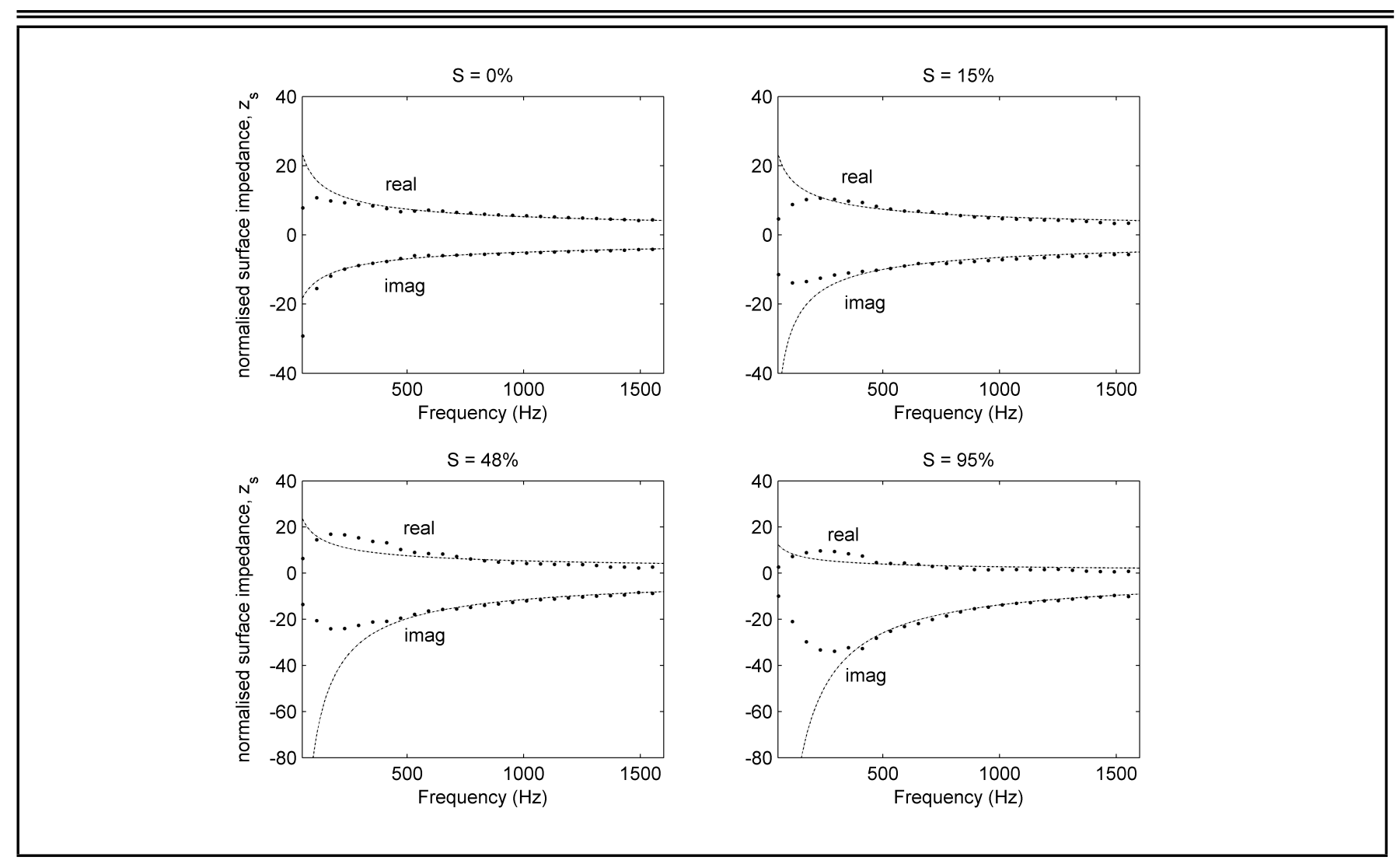

Figure 9. The measured (dots) and predicted (dashed lines) frequency dependence of the acoustic surface impedance of a $50 \mathrm{~mm}$ layer of fine silica sand.

distributed pore size. It is easy to show that the latter parameter is unimportant for modelling the acoustical properties of a highly porous material such as melamine so that this parameter was set to zero, i.e. $\sigma_{s}=0$. The fitted value of the flow resistivity is $\sigma=12.7 \mathrm{k} \mathrm{Pa} \mathrm{s} \mathrm{m}^{-2}$. It is easy to see from Fig. 10 that the fit is very close. The root mean square error between the measure and fitted impedance spectra here is only $1.3 \%$. The application of the Páde approximation model together with the absorption coefficient spectrum yields the value of the flow resistivity of $13.6 \mathrm{KPa} \mathrm{s} \mathrm{m}^{-2}$, which is $7 \%$ higher than the value inverted from the surface impedance data. It is easy to show that the application of the alternative, 1-parameter Delany and Bazley mode ${ }^{10}$ to the surface impedance data yields the value of the flow resistivity of $\sigma=11.9 \mathrm{k} \mathrm{Pa} \mathrm{s} \mathrm{m}^{-2}$, which is within $7 \%$ of the value of $\sigma=12.7 \mathrm{kPa} \mathrm{s} \mathrm{m}^{-2}$ determined with the Páde approximation. The Delany and Bazley model is empirical and it is not causal. ${ }^{47}$ ) Therefore, care should be taken when this model is applied to invert the flow resistivity from acoustical data, particularly complex impedance data because of the model limitations. In the case of partially reticulated foams, the porosity and tortuosity are no longer a unity. Therefore, the reticulation rate can be estimated through these parameters which can be inverted through the suggested optimisation procedure and relations. ${ }^{48}$

\subsection{Gravels}

Another interesting application of the direct minimisation procedure is to recover the permeability, porosity and tortuosity of gravels. This information is important because gravels are used extensively for drainage and in foundations. The ability of gravels to drain water is a key characteristic needed to

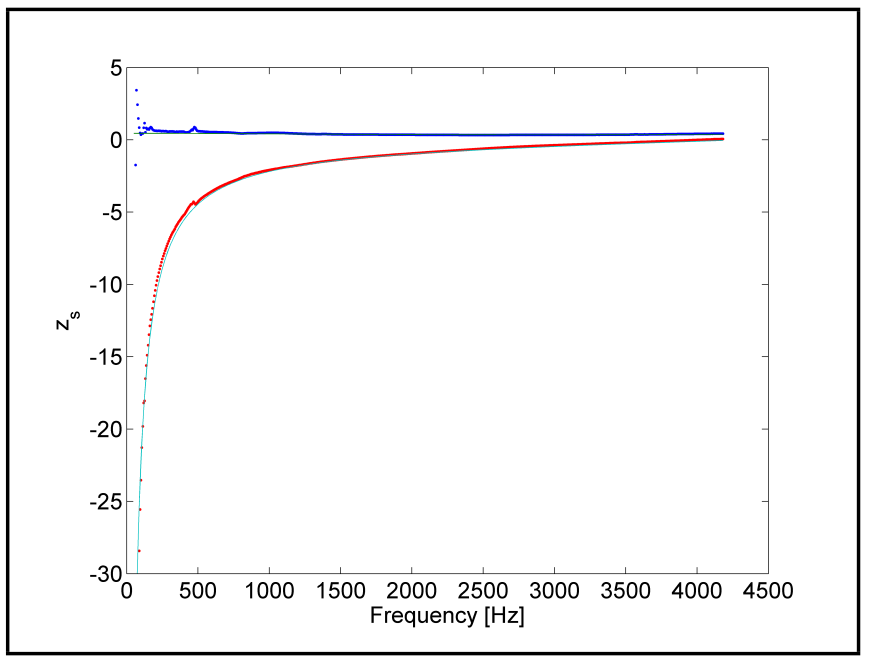

Figure 10. The measured values of the acoustic surface impedance (dots) and fitted surface impedance (solid lines) of a $17.7 \mathrm{~mm}$ thick, hard-backed layer of melamine foam.

maintain and rehabilitate critical civil engineering infrastructure where structural health depends on the ability to store and drain water. Fig. 11 presents the real and imaginary parts of the normal incidence surface impedance of a $200 \mathrm{~mm}$ thick layer of hard-backed gravel with the equivalent particle diameter in the range of 15-20 $\mathrm{mm}$ (see Fig. 12). This figure shows the measured impedance and that fitted using the 4-parameter Páde approximation model ${ }^{6}$ and 5-parameter Johnson-ChampouxAllard (JCA) model $^{502}$. We note that the two predictions are

${ }^{2}$ It is worth noting here that a common problem of many publications in which the Johnson-Champoux-Allard is presented is their liberal interpretation of this model. Some publications treat it as an equivalent fluid, whereas others as a equivalent material model. In this particular example and in many other 


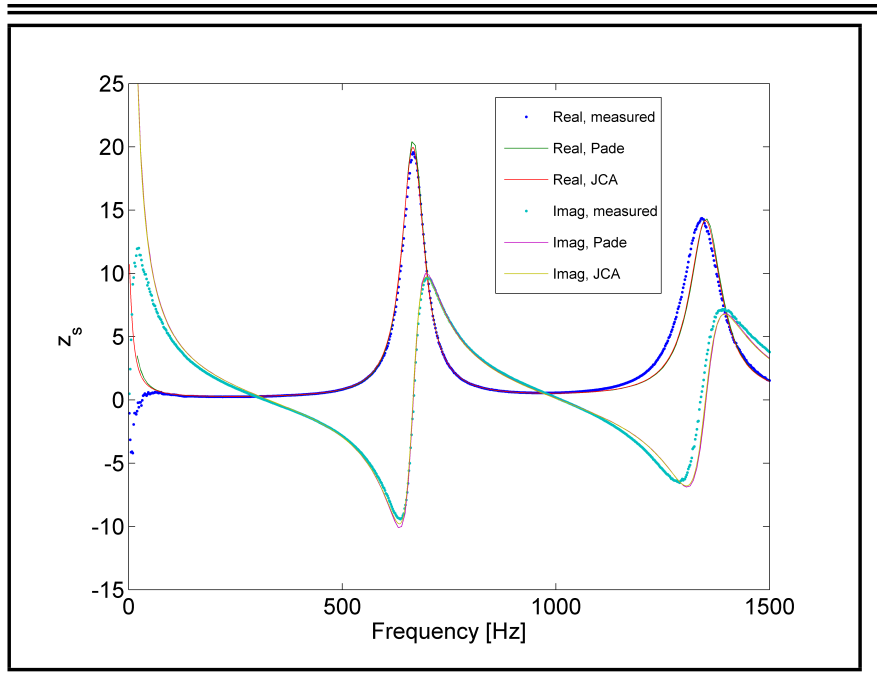

Figure 11. The measured values of the acoustic surface impedance (dots) and fitted surface impedance (solid lines) of a $200 \mathrm{~mm}$ thick, hard-backed layer of $15-22 \mathrm{~mm}$ particle gravel.

almost indistinguishable. The absolute mean error between the measured and predicted values of the surface impedance is approximately 5\%. This match is achieved with the following values of the non-acoustical parameters in the Pade approximation model: $\sigma=218 \mathrm{~Pa} \mathrm{~s} \mathrm{~m}^{-2}, \phi=0.424, \alpha_{\infty}=1.51$ and $\sigma_{s}=0.516$. The parameters in the JCA model were the same for the flow resistivity, porosity and tortuosity, but the viscous and thermal characteristic lengths were set to $\Lambda=\Lambda^{\prime}=1.24$ $\mathrm{mm}$. These values of were set equal to enable the comparison between the Pade approximation and JCA model. The Páde approximation model is for cylindrical cross-section so it does not account for the variation of the pore cross-section along the pore length. This also reduces the ambiguity in the parameter inversion process.

The non-acoustical data derived from the acoustical inversion procedure enable us to estimate the air permeability (see Eq. (23 with $\sigma_{e}=\sigma$ ) and characteristic pore dimension. For the gravel specimen considered in this work $\kappa_{p}=8.29 \times 10^{-8}$ $\mathrm{m}^{-2}$, which is approximately $10^{5}$ Dercy units. This value is close to the permeability values typical for gravels. ${ }^{49}$ The characteristic pore dimension can be estimated from eq. 18, which yields $s=1.54 \mathrm{~mm}$. It is of interest to compare the characteristic pore dimension with the viscous characteristic length. It was suggested that these two parameters are related with ${ }^{20}$

$$
\Lambda=s e^{-3 / 2\left(\sigma_{s} \ln 2\right)^{2}} .
$$

Direct substitution of the characteristic dimension $s=1.54$ $\mathrm{mm}$ and $\sigma_{s}=0.516$ gives $\Lambda=1.27 \mathrm{~mm}$, which is within $2 \%$ of the value of $\Lambda$ derived from the fitting procedure. In this case with gravel the value of the tortuosity is much higher than unity and standard deviation in the pore size is relatively large. It is of interest to estimate the value of the Pride parameter (see Eq. (6)) and low-frequency (static) tortuosity limit (see Eq. (19)). The substitution of inverted values of $\sigma_{s}$ and $\alpha_{\infty}$ in these expressions yields $\beta=1.22$ and $\alpha_{0}=3.36$, which means that $\alpha_{0}$ is 2.2 times higher than $\alpha_{\infty}$. It is also worth noting that Eq. (17) underestimates the median particle radius

practical problems of material characterisation it should be interpreted as an equivalent material model which is correctly presented in ref. ${ }^{50}$

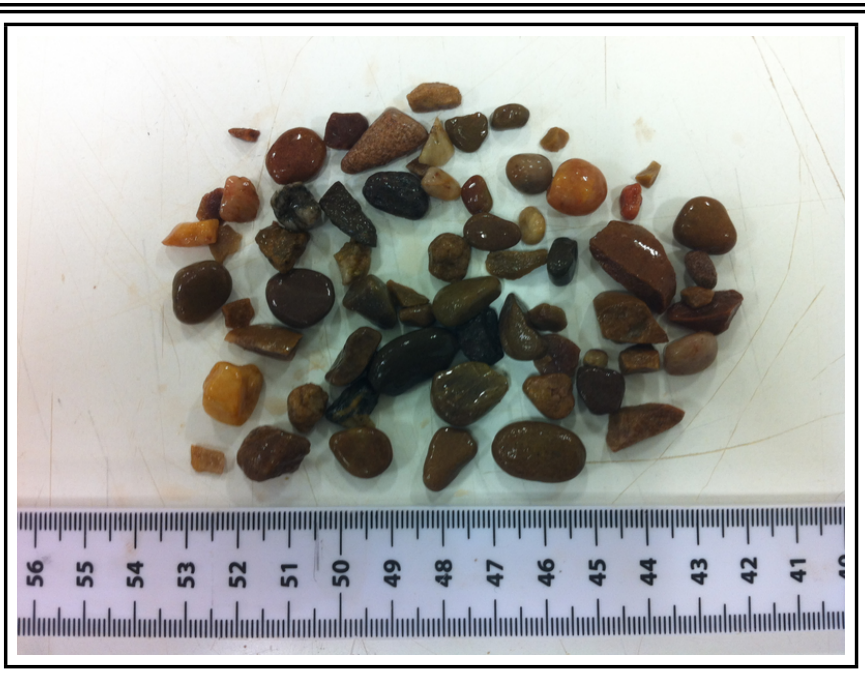

Figure 12. A photograph of gravel particles which diameter was estimated from acoustical data.

of gravel by approximately $65 \%$ because the gravel particles are not perfectly round. It is possible to improve the accuracy of this estimation within $15 \%$ if the Kuwabara/Strout model ${ }^{31}$ is adopted for the parameter $\Theta$ in Eq. (17), i.e., $\Theta=1-\phi$.

\subsection{Living Plants}

Interestingly, the parameter inversion procedure can help to determine the non-acoustical properties of living plants. ${ }^{51}$ Horoshenkov et al. used the 3-parameter Miki model ${ }^{8}$ to estimate the effective flow resistivity, tortuosity, and effective plant height from impedance tube data on the acoustical absorption coeffiecient. These parameters are important for noninvasive plant characterisation because the effective flow resistivity is directly linked to the plant leaf area density whereas the tortuosity is linked to the dominant angle of leaf orientation. ${ }^{51}$ Together with the effective plant height, these parameters can be used to estimate the living mass of green plants. In this inversion process, acoustic impedance cannot be used because of the random geometry of a living plant specimen makes it impossible to determine the exact position of the reference plane from which the distance from the top of the plant to the microphones can be accurately measured. Figure 13 shows the measured and fitted absorption coefficient spectra of a Geranium zonale plant specimen (specimen Geranium zonale-4 in Table I, in ref. ${ }^{51}$ also see Fig. 14). The fitting was achieved with the 4-parameter Páde approximation model ${ }^{6}$ and 5-parameter Johnson-Champoux-Allard (JCA) model. ${ }^{50}$ The absolute mean error between the predicted and measured absorption coefficient was within $1 \%$. The difference between the absorption spectra predicted with the two models was insignificant. The porosity was taken from Table III in ref. ${ }^{51}$ $\phi=0.98$. It was then measured directly and non-acoustically. The values of the flow resistivity, tortuosity, and standard deviation in the pore size determined with the Páde approximation model are $\sigma=36.8 \mathrm{~Pa} \mathrm{~s} \mathrm{~m}^{-2}, \alpha_{\infty}=1.90$, and $\sigma_{s}=0.587$. The values of the characteristic lengths determined with the JCA model are $\Lambda=\Lambda^{\prime}=2.10 \mathrm{~mm}$. The characteristic viscous length estimated from Eq. (25) is $\Lambda=2.19 \mathrm{~mm}$ which is within $4 \%$ with the value determined by fitting the JCA model. 


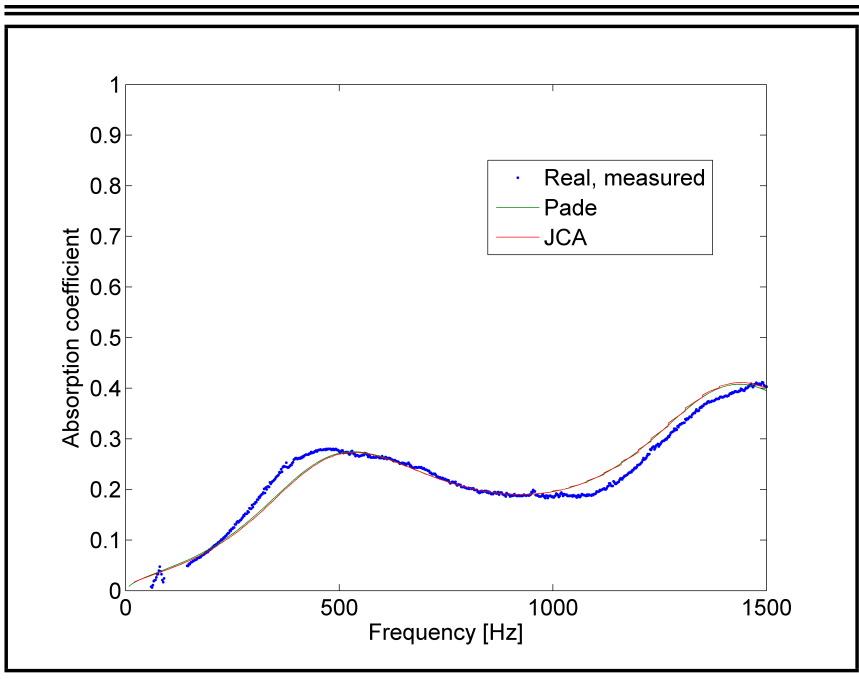

Figure 13. The measured values of the acoustic absorption coefficient (dots) and fitted absorption coefficient (solid lines) of a $130 \mathrm{~mm}$ high, hard-backed Geranium zonale plant.

\section{CONCLUSIONS}

Sound waves provide a very attractive method for noninvasive measurements of the morphological characteristics of porous materials. Therefore, the purpose of this paper has been to illustrate how acoustical models of variable complexity can be applied to determine useful non-acoustical parameters of porous media that are related to their morphological characteristics.

In the case of reticulated foams and fibrous materials with high porosity and low tortuosity, the most important nonacoustical parameter that controls the acoustical properties of these media is the flow resistivity. This parameter can be derived from the low-frequency asymptotic behaviour of the dynamic density of the equivalent fluid filling the material pores. For this type of porous media, the flow resistivity can also be derived by solving the optimisation problem whereby the difference between the predicted acoustical property (e.g., surface impedance) and measured data is minimised. These media can be modelled with a simple 1-parameter mode ${ }^{10}$ or a more complex model ${ }^{6}$ in which the values of porosity and tortuosity are set to unity.

The acoustical properties of porous media composed of particles/grains with well defined shapes can be described accurately by the flow resistivity, porosity, and tortuosity. The flow resistivity of this type of porous media can also be estimated from the low-frequency limit of the dynamic density, whereas the tortuosity can be estimated from the high-frequency behaviour of the refraction index. It is usually easy to measure the porosity of these media independently e.g., from the density ratio or geometrical considerations, or from Berryman's expression (see Eq. (16)) through the tortuosity which can be inverted acoustically. An alternative method to estimate the porosity is from the high-frequency limit of the dynamic density in which the tortuosity value is estimated from the refraction index behaviour. If a porous medium is composed of relatively small grains for which the flow resistivity is relatively high, i.e., $\omega / \sigma_{e} \ll 1$, e.g., sandy soil, then its acoustical properties can be predicted with a 2 -parameter model. ${ }^{37}$ The two parameters in this model are the effective flow resistivity and

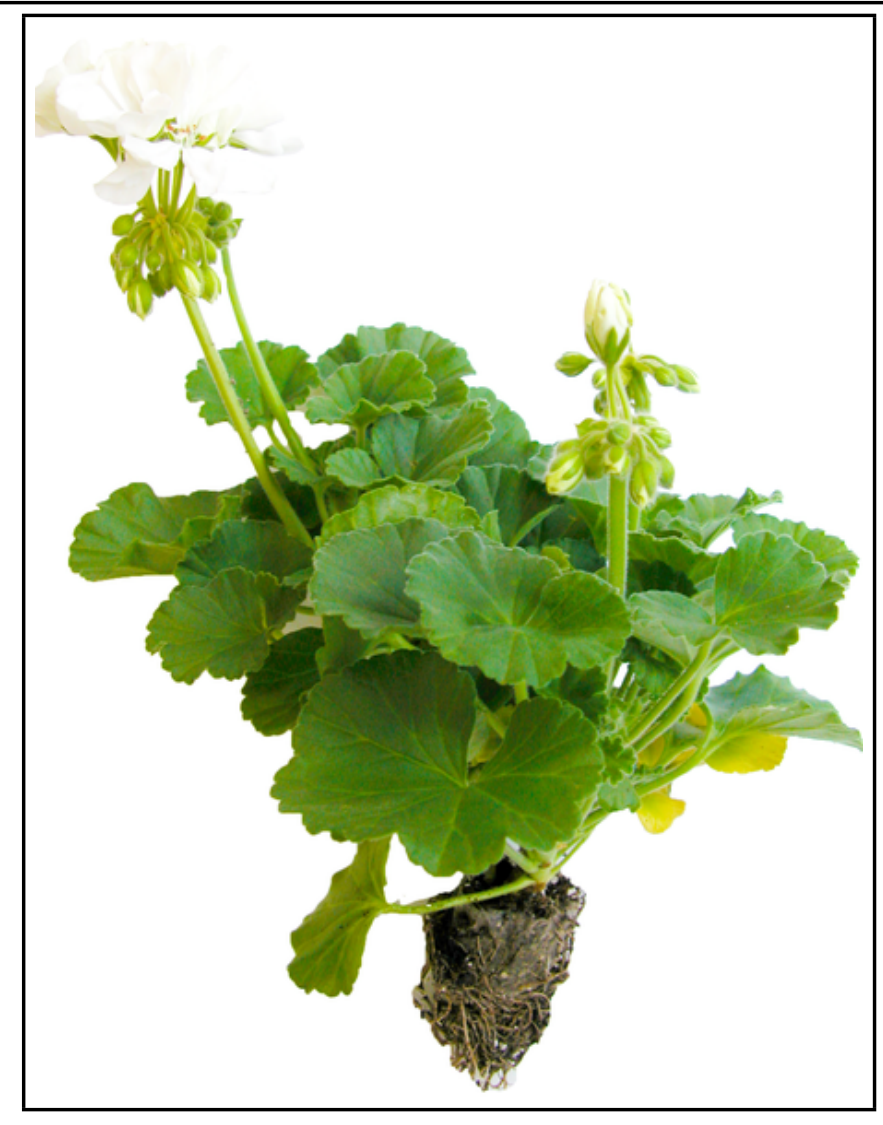

Figure 14. The leaf size of this Geranium zonale plant was estimated from acoustical data.

rate of porosity change. These can be derived from the least mean square analysis of the surface impedance measured either in the laboratory or in the field.

The acoustical properties of more complex materials, e.g., angular gravels and living plants, can be predicted using a model with 4 or more parameters, e.g., Páde approximation model $^{6}$ or JCA model. ${ }^{50}$ It has been shown that the parameters of the pore size distribution and characteristic viscous length used in these models are inter-related. More specifically, it has been confirmed from the analysis of experimental data and model behaviour that the mean pore size and standard deviation in pore size distribution in the Páde approximation model and viscous characteristic length in the JCA model are closely related via Eq. (25). This is important because both the mean pore size and standard deviation in pore size are directly measurable parameters, ${ }^{45}$ whereas the viscous characteristic length is difficult to measure directly. It has been shown that these two models are sufficiently robust to be used in the parameter inversion process, which is based on the optimisation procedure applied to the surface impedance data or using the numerical approach. ${ }^{2,39,40}$ The flow resistivity and other parameters determined from the inversion process can be used to estimate the size of particles that composed the material skeleton and size of pores, which can be important for a number of non-acoustic applications.

\section{ACKNOWLEDGEMENTS}

I would like to thank Dr. Paolo Bonfiglio, Dr. Luc Jaouen, and Professor Abigail Bristow for proofreading this paper, pro- 
viding me with very helpful comments, and pointing me to useful references that I initially overlooked. I would also like to thank Dr. Michael Pelegrinis for his optical image analysis of the sample of reticulated foam that was discussed in section 2.1. Finally, I would like to thank the referees and Dr. Malcolm Crocker for their helpful comments.

\section{REFERENCES}

1 Biot, M. A. Theory of elastic wave propagation in a fluid saturated porous solid. I. Low-frequency range, J. Acoust. Soc. Am., 28 (2), 168-178, (1956). http://dx.doi.org/10.1121/1.1908239

2 Biot, M. A. Theory of elastic wave propagation in a fluid saturated porous solid. II. Higher-frequency range, J. Acoust. Soc. Am., 28 (2), 179-191, (1956). http://dx.doi.org/10.1121/1.1908241

3 Stoll, R. D. Sediment Acoustics, Springer-Verlag, New York, (1989).

4 Embleton, T. F. W., Piercy, J. E., and Olson, N. Outdoor sound propagation over ground of finite impedance, J. Acoust. Soc. Am., 59 (2), 267-277, (1976). http://dx.doi.org/10.1121/1.380882

5 Allard, J. F. and Atalla, N. Propagation of Sound in Porous Media: Modelling of Sound Absorbing Materials, $2^{\text {nd }}$ edition, John Wiley \& Sons, Chichester, UK (2009).

6 Horoshenkov, K. V., Attenborough, K., and ChandlerWilde, S. N. Páde approximants for the acoustical properties of rigid frame porous media with pore size distribution, J. Acoust. Soc. Am., 104 (3), 1198-1209, (1998). http://dx.doi.org/10.1121/1.424328

7 ImageJ, An open platform for scientific image analysis, http://imagej.net/Welcome, last accessed on 27 March 2016.

8 Miki, Y. Acoustic properties of porous materials: Generalization of empirical models, J. Acoust. Soc. Jap. (E), 11, 25-28, (1990). http://dx.doi.org/10.1250/ast.11.25

9 Pride, S. R., Morgan, F. D., and Gangi, A. F. Drag forces in porous medium acoustics, Physical Review B, 47, 49644978, (1993). http://dx.doi.org/10.1103/physrevb.47.4964

10 Delany, M. E. and Bazley, E. N. Acoustical properties of fibrous absorbent materials, Appl. Acoust., 3, 105-116, (1970). http://dx.doi.org/10.1016/0003-682x(70)90031-9

11 ISO 10534-2:1998, Determination of sound absorption coefficient and impedance in impedance tubes, Part 2: Transfer-function method, International Organization for Standardization, Geneva, Switzerland, (1998).

12 Horoshenkov, K. V., Khan, A., Bécot, F. X., Jaouen, L., Sgard, F., Pompoli, F., Prodi, N., Bonfiglio, P., Pispola, G., Asdrubali, F., Hübelt, J., Atalla, N., Amdin, C. K., Lauriks, W., and Boeckxb, L., Reproducibility experiments on measuring acoustical properties of rigid-frame porous media (round-robin tests), J. Acoust. Soc. Am., 122(1), 345353, (2007). http://dx.doi.org/10.1121/1.2739806

13 Cummings, A. Impedance tube measurements on porous media: The effect of air gaps around the sample, J. Sound Vib., 151, 63-75, (1991). http://dx.doi.org/10.1016/0022$460 x(91) 90652-\mathrm{z}$

14 Pilon, D., Panneton, R., and Sgard, F. Behavioral criterion quantifying the effects of circumferential air gaps on porous materials in the standing wave tube, J. Acoust. Soc. Am., 116 (1), 344-356 (2004). http://dx.doi.org/10.1121/1.1756611

15 Iwase, T., Izumi, Y., and Kawabata, R. A new measuring method for sound propagation constant by using sound tube without any air back of a test material, Proc. Int. Cong. Noise Contr. Eng., Christchurch, New Zealand 1618 November, (1998).

16 Doutres, O., Salissou, Y., Atalla, N., and Panneton, R. Evaluation of the acoustic and non-acoustic properties of sound absorbing materials using a three microphone impedance tube, Appl. Acoust., 71 (6), 506-509, (2010). http://dx.doi.org/10.1016/j.apacoust.2010.01.007

17 Smith, C. D. and Parrott, T. L. Comparison of three methods for measuring acoustic properties of bulk materials, J. Acoust. Soc. Am. 74 (5), 1577-1582, (1983). http://dx.doi.org/10.1121/1.390119

18 Leclaire, P., Umnova, O., and Horoshenkov, K. V. Porosity measurement by comparison of air volumes, Rev. Sci. Instr., 74 (3), 1366-1370, (2003). http://dx.doi.org/10.1063/1.1542666

19 Bonfiglio, P. and Pompoli, F. Acoustical properties of polyurethane open cell materials: Experimental investigation and theoretical models, Proc. Int. Cong. Sound Vib., Cairns, Australia 9-12 July, (2014).

20 Horoshenkov, K. V., Dazel, O., and Groby, J. F. Asymptotic limits of some models for sound propagation in porous media and the assignment of the pore characteristic lengths, J. Acoust. Soc. Am., 139 (5), 2463-2474 (2016). http://dx.doi.org/10.1121/1.4947540

21 Wang, D., Zhang, X., Luo, S., and Li, S. Preparation and property analysis of melamine formaldehyde foam, Adv. Mat. Phys. Chem., 2, 63-67 (2012). http://dx.doi.org/10.4236/ampc.2012.24b018

22 Bonfiglio, P. and Pompoli, F. Inversion problems for determining physical parameters of porous materials: Overview and comparison between different methods, Acta Acustica united with Acustica,99 (3), 341-351, (2013). http://dx.doi.org/10.3813/aaa.918616

23 Johnson, D. L., Koplik, J., and Dashen, R. Theory of dynamic permeability and tortuosity in fluid-saturated porous media, J. Fluid Mech., 176, 379-402, (1987). http://dx.doi.org/10.1017/s0022112087000727 
24 Bies, D. A. and Hansen, C. H. Flow resistivity information for acoustical design, Appl. Acoust., 13(5), 357-391, (1980). http://dx.doi.org/10.1016/0003-682x(80)90002-x

25 Garai, M. and Pompoli, F. A simple empirical model of polyester fibre materials for acoustical applications, Appl. Acoust., 66, 1383-1398, (2005). http://dx.doi.org/10.1016/j.apacoust.2005.04.008

26 Carman, P. C. Fluid flow through granular beds, Trans. Inst. Chem. Eng., (London), 15, 150-166, (1937).

27 Zwikker, C. and Kosten, C. W. Sound Absorbing Materials, Elsevier, Amsterdam, 1949.

28 Champoux, I. and Stinson, M. R. On acoustical models for sound propagation in rigid frame porous materials and the influence of shape factors, J. Acoust. Soc. Am., 92 (2), 1120-1131, (1992).http://dx.doi.org/10.1121/1.405281

29 Berryman, J. G. Confirmation of Biot's theory, Appl. Phys. Lett., 37, 382-384, (1980). http://dx.doi.org/10.1063/1.91951

${ }^{30}$ Leclaire, P., Kelders, L., Lauriks, W., Glorieux, C., and Thoen, J. Determination of the viscous characteristic length in air-filled porous materials by ultrasonic attenuation measurements, J. Acoust. Soc. Am., 99 (4), 1944-1948, (1996). http://dx.doi.org/10.1121/1.415378

31 Umnova, O., Attenborough, K. and Li, K. Cell model calculations of dynamic drag parameters in packings of spheres, J. Acoust. Soc. Am., 107 (6), 3113-3119, (2000). http://dx.doi.org/10.1121/1.429340

32 Perrot, C., Chevillotte, F., and Panneton, R. Dynamic viscous permeability of an open cell aluminium foam: Computations versus experiments, J. Appl. Phys., 103, 024909, (2008). http://dx.doi.org/10.1063/1.2829774

33 Zuckerwar, A. J. Acoustic ground impedance meter, J. Acoust. Soc. Am., 73 (6), 2180-2186, (1983). http://dx.doi.org/10.1121/1.389542

34 Cramond, A. J. and Don, C. G., Effects of moisture content on soil impedance, J. Acoust. Soc. Am., 82, 293-301, (1987). http://dx.doi.org/10.1121/1.395565

35 ANSI S1.18-2010, American National Standard Method for Determining the Acoustic Impedance of Ground Surfaces, (2010)

36 Horoshenkov, K. V. and Mohamed, M. H. A., Experimental investigation of the effects of water saturation on the acoustic admittance of sandy soils, J. Acoust. Soc. Am., 120 (4), 1910-1921, (2006). http://dx.doi.org/10.1121/1.2338288

37 Attenborough, K., Ground parameter information for propagation modelling, J. Acoust. Soc. Am., 92, 418-427, (1992). http://dx.doi.org/10.1121/1.404251
38 Mohamed, M. A. H. and Horoshenkov, K. V. An airborne acoustic method to determine the volumetric water content of unsaturated sands, $J$. Geotech. Geoenvi. Eng., 135(12), 1872-1882, (2009). http://dx.doi.org/10.1061/(asce)gt.1943-5606.0000168

39 Panneton, R. and Olny., X. Acoustical determination of the parameters governing viscous dissipation in porous media, J. Acoust. Soc. Am., 119 (4), 2027-2040, (2006). http://dx.doi.org/10.1121/1.2828066

40 Olny, X. and Panneton, R. Acoustical determination of the parameters governing thermal dissipation in porous media, J. Acoust. Soc. Am., 123 (2), 814-824, (2008). http://dx.doi.org/10.1121/1.2828066

41 Bonfiglio, P. Advanced Experimental Techniques for the Description of Rigid Frame Porous Media, PhD Thesis, University of Ferarra, Italy, (2006).

42 Sun, W. and Yuan, Y. X. Optimization Theory and Methods: Nonlinear Programming Optimization and Its Applications, Chapter 8, Springer, New York (2006).

43 ISO 9053:2001 Acoustics - Materials for acoustical applications - Determination of airflow resistance, International Organization for Standardization, Geneva, Switzerland, (1991).

44 Nelder, J. A. and Mead, R., A simplex method for function minimization, Computer Journal, 7, 308-313, (1965).

45 Horoshenkov, K. V. and Swift, M. J., The acoustic properties of granular materials with pore size distribution close to log-normal, J. Acoust. Soc. Am., 110 (5), 2371-2378 (2001).http://dx.doi.org/10.1121/1.1408312

46 Horoshenkov, K. V. and Swiftm M. J. Acoustic properties of consolidated granular mixes, Appl. Acoust., $62 \quad$ (6), 665-690 (2001). http://dx.doi.org/10.1016/j.apacoust.2003.12.002

47 Horoshenkov, K. V. Control of Noise in City Streets, $\mathrm{PhD}$ Thesis, University off Bradford, 1996.

48 Doutres, O., Atalla, N., and Dong, K. Effect of the microstructure closed pore content on the acoustic behavior of polyurethane foams, J. Appl. Phys., 110(6):064901, (2011).http://dx.doi.org/10.1063/1.3631021

49 Verruijt A. Theory of Groundwater Flow, Macmillan Co, Pitman Press, (1970).

50 http://apmr.matelys.com/PropagationModels/ MotionlessSkeleton/JohnsonChampouxAllardModel.html, last accessed on 16 April 2015.

51 Horoshenkov, K. V., Khan, A., and Benkreira, H. Acoustic properties of low growing plants, $J$. Acoust. Soc. Am, 133 (5), 2554-2565, (2013). http://dx.doi.org/10.1121/1.4798671 